\section{WEB WATCH}

\section{One-stop trial shop}

- http://controlled-trials.com/

Researchers, physicians and patients have long demanded free access to clinical trial data while studies are in progress, rather than having to wait until the results are published. Current Controlled Trials Ltd, part of the Current Science Group of companies, has addressed this need by creating a free, international, searchable database of ongoing clinical trials. The metaRegister of controlled trials $(m R C T)$ is a valuable search engine for clinical studies that are underway in all areas of health care. Visitors can search by disease or sponsor for information on over 10,000 trials. The $m R C T$ is not a complete record of all randomized trials worldwide, but does allow visitors to search databases of a number of organizations simultaneously. For example, with one click, a user can search for prostate cancer clinical trials that are sponsored by Oxford University, GlaxoSmithKline, the Leukaemia Research Fund, the Hong Kong Health Services Research Fund, the UK's Medical Research

Council, the US Department of Veterans Affairs

Cooperative Studies

Program, and the National Institutes of Health. The site provides information on patient recruitment status, organizer contact details, reasons for trial termination, trial design and patient eligibility. Trial sponsors trying to recruit new patients can also enter information about their study. Information posted is not reviewed by Current Controlled Trials before being included in the database, and data accuracy is the responsibility of the contributor. The site does, however, have an advisory board made up of editors from a number of medical journals and executives from medical research organizations. Registrants can also receive regular updates by e-mail. Kristine Novak

VACCINES

\title{
Trapping technology
}

In police circles, entrapment is frowned upon as a means of securing an arrest. But, as reported by Akira Takashima and colleagues in the January issue of Nature Biotechnology, it could be a legitimate way of creating cancer vaccines without having to resort to costly ex vivo approaches.

A promising way of generating cancer vaccines uses dendritic cells - specialized antigen-presenting cells. But to generate an effective vaccine, they must be collected from the patient and then subjected to time-consuming and costly 'customizing' procedures - in which they are expanded and loaded with tumour antigens and/or cytokineencoding genes - before they can be re-administered to the patient. Could this lengthy ex vivo process be replaced by a simpler procedure?

Langerhans cells (LCs) - dendritic cells that reside in skin -

generally stay put unless they are induced to mature. Maturation involves acquiring receptors for chemokines such as macrophage inflammatory protein $3 \beta$ (MIP- $3 \beta$ ), allowing them to move along a chemokine gradient from the epidermis to draining lymph nodes. This response can be triggered by haptens - small molecules that are not antigenic unless they are associated with a larger molecule such as a protein. But what if a 'decoy' lymph node could be produced that diverts LCs away from their real destination to a site where they can easily be pulsed with antigen? To this aim, the authors produced ethylene-vinyl-acetate (EVA) rods that released MIP-3 $\beta$, and implanted them just under the abdominal skin of mice. Application of fluorescein isothiocyanate (FITC) - a hapten that also doubles up as a fluorescent signal for tracking LC migration — revealed that MIP-3 $\beta$ -

\section{GENOMIC INSTABILITY}

\section{A perfect mismatch}

Inactivation of the DNA

mismatch-repair machinery can drive tumorigenesis, as mutations in genes involved in cell growth and survival are not repaired. But does this mutator phenotype also mutate other repair genes to accelerate genomic instability and tumorigenesis? Sergei Malkhosyan and co-workers, reporting in the 18 December issue of Proceedings of the National Academy of Sciences, show that mutation of two mismatch-repair genes, rather than one, not only increases the mutation rate, but also changes the predominant type of mutation.

The SW48 human colon adenocarcinoma cell line lacks expression of the mismatch-repair

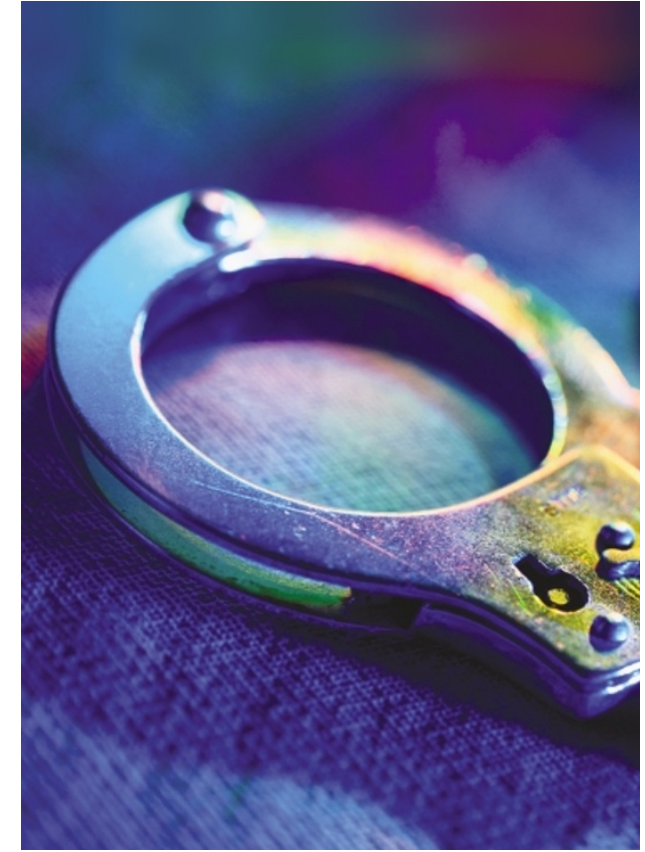

expressing rods 'trapped' LCs by attracting them to the rods. By contrast, after 24 hours, a significant number of LCs had migrated from the epidermis to the draining lymph nodes in mice with either no implanted rods or rods expressing a control protein.

So, LCs can be trapped in one location, but can they also be loaded with tumour antigens? To investigate this, rods expressing ovalbumin were implanted with those expressing MIP-3 $\beta$, and were, again, treated with a hapten. T cells harvested from the

different, depending on whether MSH6 was present. The mutations were all single point mutations, consistent with the mismatch-repair deficiency, but $\mathrm{MSH}^{+/+}$cells predominantly had single base-pair insertions or deletions, whereas $\mathrm{MSH6}^{-1-}$ cells predominantly had base substitutions and transitions, which resulted in missense or nonsense mutations.

So, mismatch repair - and the mutator phenotype that ensues when the process is deficient - is not quite as simple as we thought. The hypothesis that inactivation of $M L H 1$ would prevent all mismatch repair must be re-evaluated in light of these findings, and the idea that the mutator phenotype accelerates through a mutator 'cascade' gains further ground. The consequences of mutation in more than one mismatch-repair gene are now known, but the 


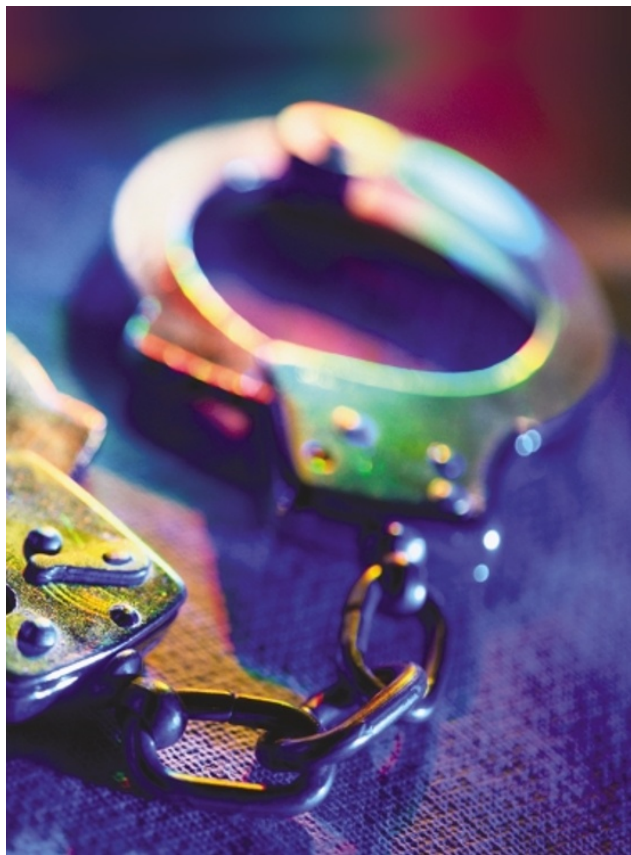

spleens of these mice five days after hapten treatment had a strong cytotoxic T-lymphocyte response against an ovalbumin-transduced cell line, and this was comparable to what could be achieved with a standard dendritic-cell vaccine.

The therapeutic and preventative efficiencies of the vaccine were also tested by inoculating mice with ovalbumin-expressing tumour cells either one day before or five days after administration of the vaccine, respectively. The vaccine had a $50-60 \%$ therapeutic efficacy, but extent of the impact that this might have on tumorigenesis remains to be determined.

Emma Greenwood

\section{(2) References and links ORIGINAL RESEARCH PAPER \\ Baranovskaya, S. et al. Functional significance of concomitant inactivation of $h M L H 1$ and}

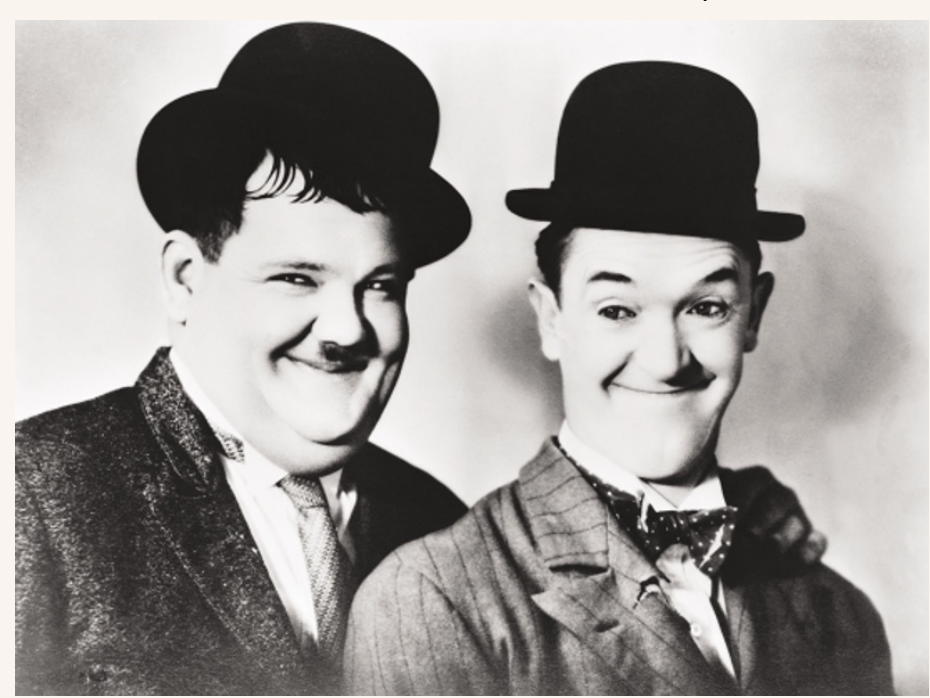

hMSH6 in tumor cells of the microsatellite mutator phenotype. Proc. Natl Acad. Sci. USA 98, 15107-15112 (2001)

\section{WEB SITES}

Sergei Malkhosyan's lab:

http://www.burnham.org/reports/5.Malkhosya n.97.html

Encyclopedia of Life Sciences:

http://www els net provided mice with almost full protection when administered before tumour inoculation.

But will this vaccine strategy also work with tumour antigens isolated from a patient's tumour an essential prerequisite for its successful translation into the clinic? The authors incorporated crude extracts from the $\mathrm{S} 1509$ a fibrosarcoma cell line into the EVA rods and co-implanted them in mice with rods expressing MIP-3 $\beta$. Following hapten treatment, the mice had cytotoxic T-lymphocyte activities that were able to lyse S1509a targets.

This anticancer vaccine strategy therefore seems to be as effective as traditional dendritic cell vaccines, but has the advantage of being in situ. Let's hope that these new technologies will soon provide real benefit to patients in the clinic.

Emma Greenwood

\section{(D) References and links} ORIGINAL RESEARCH PAPER Kumamoto, T. et al. Induction of tumor-specific protective immunity by in situ Langerhans cell vaccine. Nature Biotechnol. 20, 64-69 (2002) WEB SITE

Akira Takashima's lab: http://swnt240.swmed. edu/gradschool/webrib/takashim.htm

\section{IN BRIEF}

\section{APOPTOSIS}

Identification of BARD1 as mediator between proapoptotic stress and p53-dependent apoptosis Irminger-Finger, I. et al. Mol. Cell. 8, 1255-1266 (2001)

We know remarkably little about the function of BRCA1, but even less about its binding partner BARD1. This study indicates that BARD1 has a dual mode of action: in the presence of BRCA1, BARD1 acts as BRCA1's accomplice in DNA repair, but the authors find that it can also act independently of BRCA1 to elevate levels of $\mathrm{p} 53$ and promote apoptosis. The ratio of BRCA1 to BARD1 might therefore be involved in life or death decisions.

\section{TUMOUR SUPPRESSORS}

\section{Activation of retinoblastoma in mammary gland leads} to ductal growth suppression, differentiation, and adenocarcinoma.

Jiang, Z. \& Zacksenhaus, E. J. Cell Biol. 156, 185-198 (2002)

Although retinoblastoma is a tumour suppressor, its activation might also promote tumorigenesis because it suppresses apoptosis. Transgenic mice that expressed constitutively active $\mathrm{Rb}$ in the mammary gland showed reduced cell proliferation, premature differentiation and increased survival of epithelial cells. About $30 \%$ of these mice developed hyperplastic nodules, and $\sim 7 \%$ developed mammary adenocarcinomas. Given these results, the development of anticancer therapies that involve activating $\mathrm{Rb}$ should be reconsidered.

\section{IMMUNOTHERAPY} difficulty in obtaining sufficient numbers of these cells, the authors developed artificial antigen-presenting cells (APCs) that express ligands for the T-cell receptor and co-stimulatory surface molecules. These APCs activate and rapidly expand polyclonal or antigenspecific $\mathrm{CD}^{+} \mathrm{T}$ cells. The starting repertoire of $\mathrm{CD}^{+} \mathrm{T}$ cells was preserved during culture, and T-cell apoptosis was diminished.

\section{GENE THERAPY}

Chromosomal effects of adeno-associated virus vector integration.

Miller, D. G. et al. Nature Genet. 30, 147-148 (2002)

Adeno-associated viruses (AAVs) are currently in development as a delivery mechanism for gene therapy. But could integration of these viruses within host-cell DNA have a deleterious effect? Miller et al. now show that AAVs integrate at chromosome breaks — probably by a non-homologous end-joining pathway - and are often associated with deletions and chromosomal rearrangements, but it remains to be determined whether AAV actually causes these breaks. 